\title{
Correction to: The emerging role of probiotics as a mitigation strategy against coronavirus disease 2019 (COVID-19)
}

\author{
Rasoul Mirzaei ${ }^{1,2} \cdot$ Adeleh Attar $^{3} \cdot$ Saher Papizadeh $^{4} \cdot$ Ali Salimi Jeda $^{5} \cdot$ Seyed Reza Hosseini-Fard ${ }^{6}$. \\ Elaheh Jamasbi ${ }^{7}$. Sima Kazemi ${ }^{1}$. Saman Amerkani ${ }^{4}$. Gholam Reza Talei ${ }^{8}$. Pouya Moradi ${ }^{9}$. Saba Jalalifar ${ }^{10}$. \\ Rasoul Yousefimashouf ${ }^{1,11} \cdot$ Mohammad Akhter Hossain $^{12} \cdot$ Hossein Keyvani ${ }^{5,13}$. Sajad Karampoor ${ }^{5,13}$ (1)
}

Published online: 12 May 2021

(c) Springer-Verlag GmbH Austria, part of Springer Nature 2021

\section{Correction to: Archives of Virology https://doi.org/10.1007/s00705-021-05036-8}

Authors would like to correct the error in their publication.

The original article has been corrected.

1. Reference 17 is incorrect. The correct one should be "The probiotic Bifidobacterium in the management of Coronavirus: A theoretical basis" https://doi.org/10. 1177/2058738420961304.

2. The unnecessary symbol '??' found in text is deleted.

Publisher's Note Springer Nature remains neutral with regard to jurisdictional claims in published maps and institutional affiliations.

The original article can be found online at https://doi.org/10.1007/ s00705-021-05036-8.

Mohammad Akhter Hossain

akhter.hossain1980@gmail.com

$\triangle$ Hossein Keyvani

keyvani.h@iums.ac.ir

$\triangle$ Sajad Karampoor sajadkarampour1987@gmail.com

1 Department of Microbiology, School of Medicine, Hamadan University of Medical Sciences, Hamadan, Iran

2 Student Research Committee, Hamadan University of Medical Sciences, Hamadan, Iran

3 Department of Microbiology and Virology, School of Medicine, Zanjan University of Medical Sciences, Zanjan, Iran

4 Department of Microbiology and Virology, School of Medicine, Kerman University of Medical Sciences, Kerman, Iran

5 Department of Virology, School of Medicine, Iran University of Medical Sciences, Tehran, Iran
6 Department of Clinical Biochemistry, School of Medicine, Tehran University of Medical Sciences, Tehran, Iran

7 Department of Anatomical Sciences, Kermanshah University of Medical Science, Kermanshah, Iran

8 Department of Virology, School of Medicine, Lorestan University of Medical Sciences, Khorramabad, Lorestan, Iran

9 Department of Virology, Faculty of Medical Sciences, Tarbiat Modares University, Tehran, Iran

10 Department of Microbiology, School of Medicine, Iran University of Medical Sciences, Tehran, Iran

11 Research Center for Molecular Medicine, Hamadan University of Medical Sciences, Hamadan, Iran

12 The Florey University of Neuroscience and Mental Health, University of Melbourne, Melbourne, VIC 3010, Australia

13 Gastrointestinal and Liver Diseases Research Center, Iran University of Medical Sciences, Tehran, Iran 